\title{
A panel of recombinant proteins from human-infective Plasmodium species for serological surveillance
}

\author{
Nicole Müller-Sienerth ${ }^{1 \dagger}$, Jarrod Shilts ${ }^{1 \dagger}$, Khamisah Abdul Kadir ${ }^{2}$, Victor Yman ${ }^{3}$, Manijeh Vafa Homann ${ }^{3}$, \\ Muhammad Asghar ${ }^{3}$, Billy Ngasala ${ }^{4,5}$, Balbir Singh ${ }^{2}$, Anna Färnert ${ }^{3,6}$ and Gavin J. Wright ${ }^{1^{*} \text { (D) }}$
}

\begin{abstract}
Background: Malaria remains a global health problem and accurate surveillance of Plasmodium parasites that are responsible for this disease is required to guide the most effective distribution of control measures. Serological surveillance will be particularly important in areas of low or periodic transmission because patient antibody responses can provide a measure of historical exposure. While methods for detecting host antibody responses to Plasmodium falciparum and Plasmodium vivax are well established, development of serological assays for Plasmodium knowlesi, Plasmodium ovale and Plasmodium malariae have been inhibited by a lack of immunodiagnostic candidates due to the limited availability of genomic information.
\end{abstract}

Methods: Using the recently completed genome sequences from P. malariae, P. ovale and P. knowlesi, a set of 33 candidate cell surface and secreted blood-stage antigens was selected and expressed in a recombinant form using a mammalian expression system. These proteins were added to an existing panel of antigens from P. falciparum and P. vivax and the immunoreactivity of $\mathrm{IgG}, \mathrm{IgM}$ and $\mathrm{IgA}$ immunoglobulins from individuals diagnosed with infections to each of the five different Plasmodium species was evaluated by ELISA. Logistic regression modelling was used to quantify the ability of the responses to determine prior exposure to the different Plasmodium species.

Results: Using sera from European travellers with diagnosed Plasmodium infections, antigens showing speciesspecific immunoreactivity were identified to select a panel of 22 proteins from five Plasmodium species for serological profiling. The immunoreactivity to the antigens in the panel of sera taken from travellers and individuals living in malaria-endemic regions with diagnosed infections showed moderate power to predict infections by each species, including P. ovale, P. malariae and P. knowlesi. Using a larger set of patient samples and logistic regression modelling it was shown that exposure to $P$. knowlesi could be accurately detected (AUC $=91 \%)$ using an antigen panel consisting of the P. knowlesi orthologues of MSP10, P12 and P38.

Conclusions: Using the recent availability of genome sequences to all human-infective Plasmodium spp. parasites and a method of expressing Plasmodium proteins in a secreted functional form, an antigen panel has been compiled that will be useful to determine exposure to these parasites.

Keywords: Plasmodium, Serology, Antigen, Recombinant protein, Antibody, Malaria

*Correspondence: gw2@sanger.ac.uk

${ }^{\dagger}$ Nicole Müller-Sienerth and Jarrod Shilts contributed equally to this work

${ }^{1}$ Cell Surface Signalling Laboratory, Wellcome Sanger Institute, Cambridge, UK

Full list of author information is available at the end of the article

\section{Background}

Malaria is an infectious disease that remains a global health problem causing an estimated 219 million clinical cases resulting in 435,000 deaths in 2017 [1]. The disease 
is caused by parasites of the genus Plasmodium and several species are known to regularly infect humans. The vast majority of deaths occur in sub-Saharan Africa and are caused by Plasmodium falciparum, whereas outside of Africa, Plasmodium vivax is responsible for over half of all malaria infections leading to significant morbidity and mortality [2]. Much less is known about the other human-infective Plasmodium species, Plasmodium malariae, Plasmodium ovale and Plasmodium knowlesi both in terms of their global distribution and clinical impact. Plasmodium knowlesi, a parasite typically found in macaques, is a significant cause of human malaria in Southeast Asia, causing a spectrum of disease ranging from mild to fatal infections [3]. Malaysia has the highest incidence of $P$. knowlesi malaria with over 6700 cases reported in the last 2 years compared to only 85 cases of indigenous human malaria (unpublished data from the Ministry of Health, Malaysia).

Diagnosis of Plasmodium infections and epidemiological surveillance is important for guiding the distribution of resources into intervention measures and establishing their clinical impact over time [4]. Methods to measure the prevalence of Plasmodium infections include microscopy, rapid diagnostic tests (RDTs) and PCR-based approaches, each differing in their sensitivity, infrastructure requirements, and ability to diagnose the different species. Serological assays can provide a historical record of infection and because of the specificity of antibody-antigen binding, could also potentially discriminate between different Plasmodium spp. infections. Host antibodies appear rapidly after initial infection and can persist for months and even years after the parasites have been cleared $[5,6]$. Serological screening has been applied in epidemiological settings to detect parasite exposure, evaluate transmission trends of malaria [7-10], and identify antibody-based correlates of protection [11, 12]. It is also used in blood donation centres, where, due to the increase in international travel and migration, the need for serological diagnosis is becoming more important to reduce the risk of transfusion-transmitted infections. Currently, many centres assess these risks using patient questionnaires which is generally unsatisfactory; moreover, the limitations and costs of the currently available serological tests often make implementing these assays economically unattractive [13].

Many antibodies recognise epitopes that are only formed in the context of an antigen in its native conformation [14]. To detect these antibodies, it is vitally important that the proteins used are correctly folded so that they faithfully form these epitopes. Expressing Plasmodium proteins in a soluble recombinant form has proved challenging, perhaps because of the high A:T content of the genome and lack of recognisable protein domains in many Plasmodium proteins [15]. This problem is especially acute for parasite proteins that are secreted or embedded in membranes because these proteins additionally contain structurally critical posttranslational modifications, such as disulfide bonds that are not typically added by many commonly used expression systems. Recently, a method of expressing large panels of recombinant Plasmodium proteins was developed that retained many of their biochemical functions [16]. Central to this approach was the use of a mammalian expression system which increases the chances that appropriate post-translational modifications are correctly added to ensure proteins adopt their native fold. For antigens expressed using this method a large fraction-and in some cases all-the immunoreactivity to antigens was heat-labile, demonstrating that antibodies that recognise conformational epitopes represent a major component of the humoral response [16]. Previously, this approach was used to create libraries of soluble recombinant merozoite cell surface and secreted proteins that encompass the entire ectodomain from both $P$. falciparum $[16,17]$ and $P$. vivax [18]. Using sera from patients living in endemic regions, several of these proteins were found to be highly immunoreactive and might therefore be useful target antigens in serological assays $[8,12]$. Expanding the antigen panel to include the other parasite species infecting humans would be especially valuable if they could be used to determine exposure to the different species of Plasmodium. Here, the recent availability of high-quality genome sequences from the three other human-infective Plasmodium parasites: P. knowlesi, P. ovale and P. malariae were used to extend the available panel of proteins and were tested for reactivity to sera from individuals infected with different Plasmodium parasites.

\section{Methods}

\section{Study populations}

Collection of sera from Malawian adults that were previously used to determine its effectiveness as an adjunct therapy to treat cerebral malaria was approved by the National Health Sciences Research Committee of Malawi [19]. Plasma from adult travellers, microscopy-diagnosed with malaria and species further confirmed by multiplex PCR for all species except $P$. knowlesi [20] after returning from visits to malaria-endemic regions were obtained from the Karolinska University Hospital, Stockholm, Sweden $(n=81)$. Of these, 53 were from travellers of European origin and 28 from travellers born in malariaendemic regions as follows: Angola (3 individuals), Burundi (1), Cameroon (1), Democratic Republic of the Congo (1), Eritrea (4), Ethiopia (1), The Gambia (1), India (3), Ivory Coast (2), Kenya (5), Pakistan (1), Thailand (1), and Uganda (4). Plasma from an endemic region with 
diagnosed infections were from a longitudinally followed population from Nyamisati, in the Rufiji region, Tanzania $(n=21)$ in 1994 when transmission was high [21]. Swedish residents with no history of visiting malaria-endemic countries were included as controls $(n=28)$. Ethical approval was granted by the Ethical Review Board of the National Institute for Medical Research in Tanzania, and the Regional Ethical Review Board in Stockholm, Sweden (Dnr. 00-084, 2012/1151-32, 2006/893-31/4, 2018/235432). Serum samples from adult malaria patients with PCR-confirmed $P$. knowlesi mono-infection were collected at Kapit Hospital in Malaysian Borneo $(n=50)$ and from uninfected adult controls from Kapit Division $(n=66)$ after informed consent had been obtained. Approval to conduct this study was obtained from the Medical Research and Ethics Committee of the Ministry of Health, Malaysia and the Medical Ethics Committee, Faculty of Medicine and Health Sciences, Universiti Malaysia Sarawak.

\section{Recombinant protein construct design, expression and manipulation}

The orthologues of immunoreactive blood-stage antigens from $P$. knowlesi, $P$. malariae and $P$. ovale were identified from their respective genome sequences [22-24]. For $P$. ovale, proteins from Plasmodium ovale curtisi were selected since the manual gene annotation of this genome resulted in complete open reading frames compared to the draft genome available for Plasmodium ovale wallikeri [24]. Sequences corresponding to the entire ectodomains were identified using software tools to predict the location of the signal peptides, GPI-anchor and transmembrane regions $[25,26]$. In some cases, for example, $P$. malariae P38, this analysis helped improve automated gene prediction by identifying missing signal peptides. Based on these predictions, the ectodomain regions were determined by removing signal sequences and transmembrane domains. All potential N-linked glycosylation sites were systematically mutated by substituting the serine/threonine in the context of an N-linked glycosylation sequon for alanine to prevent inappropriate glycosylation when expressed in mammalian cells as described previously [16]. All sequences were codonoptimized for expression in human cells, flanked with unique $5^{\prime}$ NotI and $3^{\prime}$ AscI restriction enzyme sites to allow inframe cloning in a plasmid containing a highly efficient mouse variable $\kappa$ light chain signal peptide [27] and a rat $\mathrm{Cd} 4 \mathrm{~d} 3+4$ epitope tag followed by either a peptide sequence allowing enzymatic biotinylation and/or 6-his tag for purification [28]. Proteins were expressed by transient transfection in suspension-grown HEK293E [29] and HEK293-6E cells [30], essentially as described previously [31]. In brief, HEK293 cells were seeded the day prior to transfection at a density of $2.5 \times 10^{5}$ cells $\mathrm{mL}^{-1}$ (HEK293E) or $1.0 \times 10^{6}$ cells $\mathrm{mL}^{-1}$ (HEK293-6E). Cells are routinely cultured in volumes of $50 \mathrm{~mL}$ in Freestyle293 media following the manufacturer's recommendations; for HEK293E cells, the media is supplemented with 1\% FCS. To ensure efficient biotinylation, the cell culture media used to produce bait proteins with D-biotin to a final concentration of $100 \mu \mathrm{M}$. The following day, cells were transfected as described [31] using either $25 \mu \mathrm{g}$ (HEK293E) or $50 \mu \mathrm{g}$ (HEK293-6E) of the bait plasmid constructs. To enzymatically monobiotinylate proteins, cells were co-transfected with a plasmid encoding a secreted version of the Escherichia coli BirA enzyme (Addgene plasmid number 64395), at a 10:1 ratio, essentially as described [31]. Cultures were harvested 6 (HEK293E) or 5 (HEK293-6E) days post-transfection by first pelleting the cells by centrifugation at $3000 \times g$ for 20 min followed by filtration of the supernatant through a $0.22 \mu \mathrm{M}$ filter. His-tagged proteins were purified from spent tissue culture supernatant with either a $1 \mathrm{~mL}$ HisTrap HP column (GE Healthcare) using an ÄKTAxpress or ÄKTApure instrument (GE Healthcare) or a bespoke purification instrument for parallel protein purification [28] in a His MultiTrap HP 96-well plate (GE Healthcare).

\section{Western blotting}

To determine protein integrity, $10 \mu \mathrm{L}$ of transfection supernatant was resolved by SDS-PAGE using Novex NuPAGE 4-12\% Bis Tris precast gels (Life Technologies) under reducing conditions, transferred to nitrocellulose membrane (Invitrogen), blocked with $2 \%$ BSA in phosphate-buffered saline (PBS) $/ 0.1 \%$ Tween-20 (PBST), and probed with $0.02 \mu \mathrm{g} / \mathrm{mL}$ of streptavidin-HRP (Jackson Immunoresearch) diluted in PBS-2\% BSA. After washing, biotinylated proteins were detected by addition of SuperSignal West Pico Chemiluminescent substrate (PIERCE) and developed on photographic film (Amersham Hyperfilm ECL, GE Healthcare).

\section{Enzyme-linked immunosorbent assay (ELISA)}

ELISAs were performed by capturing biotinylated bait proteins into individual wells of streptavidin-coated 384-well plates (Greiner Bio-one). Plates were washed for $30 \mathrm{~min}$ with $50 \mu \mathrm{L}$ PBS-T ( $0.2 \%$ Tween) and blocked with PBS-2\% BSA for a minimum of $3 \mathrm{~h} .20 \mu \mathrm{L}$ of a bait protein diluted in PBS- $2 \%$ BSA at a concentration previously determined as the amount required to saturate the biotin binding capacity of the well were added in triplicate and incubated for at least $16 \mathrm{~h}$ at $4{ }^{\circ} \mathrm{C}$. Antisera were centrifuged at 13,000 rpm for a minimum of $1 \mathrm{~h}$ at $4{ }^{\circ} \mathrm{C}$, diluted in PBS-2\% BSA and incubated with rotation for at least $16 \mathrm{~h}$ at $4{ }^{\circ} \mathrm{C}$ before adding to the antigencoated plates for $1 \mathrm{~h}$. Serum dilutions used were: native 
Tanzanians 1:5000, native Malaysians 1:1000, imported malaria 1:500-1:1000, European travellers 1:100-1:500, and Malawian pooled sera resuspended to $20 \mathrm{mg} \mathrm{mL}^{-1}$ and used 1:1000. Plates were washed $3 \times$ in PBS-T before incubating with 1:10,000 dilution of peroxidase-conjugated AffiniPure goat anti-human IgA + IgG + IgM $(\mathrm{H}+\mathrm{L})$ (Jackson ImmunoResearch) in PBS-2\%BSA for $1 \mathrm{~h}$. Plates were washed in PBS-T and the HRP substrate ABTS (KPL) was added and absorption at $405 \mathrm{~nm}$ determined using an automated plate reader (FluoStar Optima, BMG Labtech).

\section{Data analysis}

Data are available in Additional file 1 and all data analysis was performed in R (version 3.5.2). Background signals from a negative control well were subtracted from ELISA absorbance data, and because there was little signal to most antigens for each patient, the median across all antigens was used as a robust measure of background immunoreactivity. For cross-reactivity analysis, pairwise Spearman correlations between each measured antigen were calculated across patient sera. Immunoreactivity values that were negative after background subtraction were rounded to zero to avoid correlating uninformative negative signals. Significance tests for each rank correlation were corrected for multiple-testing using the Benjamini-Hochberg procedure. Based on the results of the cross-reactivity analysis, all antigens with significantly correlated immunoreactivity across different species were excluded from further analysis $(P f \mathrm{HPzz}, P v \mathrm{HPzz}$, PmP41, PmMSP5, PvMSP5). Logistic regression models to predict exposure were computed using the generalized linear models "glm" function in R. One model was fitted per species, making a total of five classifiers. For every model, each patient was designated as either: diagnosed for that particular species (either by qPCR or microscopy); a negative control from a non-endemic region with no infection history; or, as ambiguous, if the patient lived in an endemic region, but was not diagnosed for that particular species. Ambiguous cases were omitted in model training, since they could not be classified reliably. Once labelled, the data sets were randomly divided approximately 50:50 into a testing set and training set. Logistic regression models were fit to the training set, then evaluated on the testing sets. Receiver operating characteristic (ROC) curves were calculated using the model predictions on the testing set and their known diagnosed labels. Curves were coloured by an arbitrary cost function, summing the number of false positives and false negatives at each threshold to highlight the relative optima [32, 33]. The area under the curve (AUC) was calculated using the PRROC package [34]. To determine a confidenceinterval for the ROC curve estimates, the model fitting and evaluation process was repeated for 10 different random splits of the data into training and testing sets. Average true positive and false positive rates were calculated at each threshold, along with a 95\% confidence interval from the 10 stratifications. When calculating model scores across all patients for every species the same procedure was followed except 100 instead of 10 random sub-samplings were used to ensure that every patient was included on at least one testing set after random splitting.

\section{Results}

With the aim of identifying antigens that could be used for serological markers of infection for Plasmodium parasites that infect humans, 12 proteins from $P$. falciparum and 10 proteins from $P$. vivax which were previously shown to be highly immunoreactive to sera from patients living in endemic regions were selected $[12,18]$ (Table 1). From these proteins, 8 that were produced at high levels in the expression system (CyRPA, GAMA, MSP10, MSP4, MSP5, P12, P38, P41) were selected to identify the orthologous proteins from the genome sequences of $P$. knowlesi [22], P. ovale and P. malariae [23, 24] (Table 1). The P92 orthologue from $P$. knowlesi and 2 paralogues from both the MSP3 and MSP7 multigene family from $P$. ovale and $P$. malariae were also selected since orthologues of these proteins are highly immunoreactive in $P$. falciparum (Table 1). Protein expression plasmids encoding the entire ectodomains for the selected genes were made by gene synthesis, and proteins were expressed as soluble recombinant proteins by transfecting HEK293 cells. As expected, protein expression levels varied considerably, and most were expressed at usable levels at the expected size (Fig. 1a, Table 1). Exceptions included the P38 orthologue from all 3 species, P12 from P. malariae and $P$. ovale, and $P m$ CyRPA, which were all repeatedly expressed at low levels in independent transfections. PoMSP3.5, PoMSP7.8 and PkP41 showed evidence of some proteolytic processing (Fig. 1a). In summary, orthologues of proteins in the genomes of $P$. knowlesi, $P$. ovale and $P$. malariae that are highly immunoreactive to sera from patients with $P$. falciparum and $P$. vivax infections were identified and expressed as soluble recombinant proteins for serological screening.

A smaller subset within the expanded panel of recombinant proteins which were immunoreactive needed to be identified as suitable serological markers of infection. To determine which of the $P$. ovale and $P$. malariae proteins were immunoreactive, the responses to IgG, IgM and IgA immunoglobulins were tested using sera pooled from over 800 adults living in Malawi [19] where there is active transmission for both species [35]. For P. malariae, P41, MSP5, and MSP10 were selected as they were the most immunoreactive and expressed at acceptable 
Table 1 Details of the Plasmodium spp. proteins expressed in this study

\begin{tabular}{|c|c|c|c|c|c|c|c|c|}
\hline Sp. & $\begin{array}{l}\text { Official } \\
\text { nomenclature }\end{array}$ & Synonym/s & Accession number & $\begin{array}{l}\text { Region } \\
\text { expressed }\end{array}$ & $\begin{array}{l}\text { Length } \\
\text { (aa) }\end{array}$ & $\begin{array}{l}\text { Exp. } \\
\text { level }\end{array}$ & PlasmoDB, previous ID & $\begin{array}{l}\text { Addgene } \\
\text { ID }\end{array}$ \\
\hline \multirow[t]{13}{*}{ P. falciparum } & $\mathrm{MSP}^{*}$ & $\begin{array}{l}\text { MSA1, Pf190, } \\
\text { Pf195 }\end{array}$ & PF3D7_0930300 & V20-S1701 & 1682 & Low & $\begin{array}{l}\text { Merozoite surface } \\
\text { protein } 1\end{array}$ & 47709 \\
\hline & $\mathrm{AMA} 1^{*}$ & $\begin{array}{l}\text { Pf83, RMA1, } \\
\text { PfAMA1 }\end{array}$ & PF3D7_1133400 & Q25-T541 & 517 & High & $\begin{array}{l}\text { Apical membrane anti- } \\
\text { gen 1,PF11_0344 }\end{array}$ & 47741 \\
\hline & P92* & Pf92 & PF3D7_1364100 & A26-S770 & 745 & Low & $\begin{array}{l}\text { 6-cysteine protein, } \\
\text { PF13_0338 }\end{array}$ & 47728 \\
\hline & MSP4 & & PF3D7_0207000 & Y29-S253 & 225 & Low & $\begin{array}{l}\text { Merozoite surface pro- } \\
\text { tein 4, PFB0310C }\end{array}$ & 47717 \\
\hline & MSP5 & & PF3D7_0206900.1 & N22-S251 & 230 & Low & $\begin{array}{l}\text { Merozoite surface pro- } \\
\text { tein 5, PFB0305C }\end{array}$ & 47718 \\
\hline & $\mathrm{P} 12^{*}$ & Pf12, Pfs12 & PF3D7_0612700 & $\mathrm{H} 26-\mathrm{S} 323$ & 298 & High & $\begin{array}{l}\text { 6-cysteine protein, } \\
\text { MAL6P1, PFF0615C }\end{array}$ & 47725 \\
\hline & GAMA & PSOP9 & PF3D7_0828800 & L22-P710 & 689 & Med & $\begin{array}{l}\text { GPI-anchored microne- } \\
\text { mal antigen }\end{array}$ & 47747 \\
\hline & MSP10* & & PF3D7_0620400 & $\mathrm{H} 27-\mathrm{S} 503$ & 477 & Med & $\begin{array}{l}\text { Merozoite surface pro- } \\
\text { tein 10, MAL6P1.221, } \\
\text { PFF0995C }\end{array}$ & 47719 \\
\hline & P38 & Pf38, Pfs38 & PF3D7_0508000 & Q22-S328 & 307 & High & $\begin{array}{l}\text { 6-cysteine protein, } \\
\text { PFE0395C }\end{array}$ & 47727 \\
\hline & MSP3.1* & MSP3, SPAM & PF3D7_1035400 & $\mathrm{K} 26-\mathrm{H} 354$ & 328 & Med & $\begin{array}{l}\text { Merozoite surface pro- } \\
\text { tein 3,PF10_0345 }\end{array}$ & 47731 \\
\hline & P41 & Pf41, Pfs41 & PF3D7_0404900 & $\mathrm{K} 21-\mathrm{S} 378$ & 358 & High & $\begin{array}{l}\text { 6-cysteine protein, } \\
\text { PFD0240C }\end{array}$ & 47739 \\
\hline & MSP7 & & PF3D7_1335100 & T28-M351 & 324 & Med & $\begin{array}{c}\text { Merozoite surface pro- } \\
\text { tein 7,PF13_0197 }\end{array}$ & 47735 \\
\hline & $H P z z^{*}$ & PFA0210c & PF3D7_0104200 & Y24-D466 & 443 & Low & $\begin{array}{l}\text { StAR-related lipid trans- } \\
\text { fer protein, MAL1P1.33 }\end{array}$ & 50821 \\
\hline \multirow[t]{10}{*}{ P. vivax } & MSP4 & & PVX_003775 & $\mathrm{A} 26-\mathrm{S} 227$ & 202 & Low & $\begin{array}{l}\text { Merozoite surface } \\
\text { protein 4, putative, } \\
\text { PV003775 }\end{array}$ & 68510 \\
\hline & MSP5* & & PVX_003770 & $\mathrm{R} 22-\mathrm{S} 367$ & 346 & Med & $\begin{array}{l}\text { Merozoite surface pro- } \\
\text { tein 5, PV003770 }\end{array}$ & 68511 \\
\hline & P12* & & PVX_113775 & F24-A339 & 316 & Med & $\begin{array}{l}\text { 6-cysteine protein, } \\
\text { PV113775 }\end{array}$ & 68516 \\
\hline & GAMA & & PVX_088910 & L21-S749 & 729 & Med & $\begin{array}{l}\text { GPI-anchored microne- } \\
\text { mal antigen, putative, } \\
\text { PV088910 }\end{array}$ & 68522 \\
\hline & CyRPA & & PVX_090240 & T23-D366 & 344 & Med & $\begin{array}{l}\text { Cysteine-rich protec- } \\
\text { tive antigen, putative, } \\
\text { PV090240 }\end{array}$ & 68525 \\
\hline & $H P z z^{*}$ & & PVX_081550 & R23-F495 & 473 & Low & $\begin{array}{l}\text { StAR-related lipid trans- } \\
\text { fer protein, putative, } \\
\text { PV081550 }\end{array}$ & 68532 \\
\hline & P38* & & PVX_097960 & K29-G334 & 306 & Med & $\begin{array}{l}\text { 6-cysteine protein, } \\
\text { PV097960 }\end{array}$ & 68518 \\
\hline & MSP7.1* & & PVX_082700 & E22-Y420 & 399 & Med & $\begin{array}{l}\text { Merozoite surface } \\
\text { protein } 7 \text { (MSP7), } \\
\text { PV082700 }\end{array}$ & 68512 \\
\hline & MSP3Y & MSP3.1 & PVX_097670 & N21-K845 & 825 & Med & $\begin{array}{l}\text { Merozoite surface pro- } \\
\text { tein 3, PV097670 }\end{array}$ & 68506 \\
\hline & $\mathrm{P} 41^{*}$ & & PVX_000995 & E22-E384 & 363 & Med & $\begin{array}{l}\text { 6-cysteine protein, } \\
\text { PV000995 }\end{array}$ & 68519 \\
\hline
\end{tabular}


Table 1 (continued)

\begin{tabular}{|c|c|c|c|c|c|c|c|c|}
\hline Sp. & $\begin{array}{l}\text { Official } \\
\text { nomenclature }\end{array}$ & Synonym/s & Accession number & $\begin{array}{l}\text { Region } \\
\text { expressed }\end{array}$ & $\begin{array}{l}\text { Length } \\
\text { (aa) }\end{array}$ & $\begin{array}{l}\text { Exp. } \\
\text { level }\end{array}$ & PlasmoDB, previous ID & $\begin{array}{l}\text { Addgene } \\
\text { ID }\end{array}$ \\
\hline \multirow[t]{12}{*}{ P. malariae } & CyRPA & & PmUG01_05040800 & E20-D358 & 339 & Low & $\begin{array}{l}\text { Cysteine-rich protective } \\
\text { antigen, putative }\end{array}$ & 126817 \\
\hline & GAMA & & PmUG01_05017200 & L22-S764 & 743 & High & $\begin{array}{l}\text { GPl-anchored microne- } \\
\text { mal antigen }\end{array}$ & 126818 \\
\hline & MSP10* & & PmUG01_11043200 & N27-S389 & 363 & Low & $\begin{array}{l}\text { Merozoite surface pro- } \\
\text { tein } 10, \text { putative }\end{array}$ & 126819 \\
\hline & MSP4 & & PmUG01_04025600 & Y29-S206 & 178 & Low & $\begin{array}{l}\text { Merozoite surface } \\
\text { protein 4, putative }\end{array}$ & 126820 \\
\hline & MSP5* & & PmUG01_04025700 & K22-L291 & 270 & High & $\begin{array}{l}\text { Merozoite surface } \\
\text { protein } 5\end{array}$ & 126821 \\
\hline & P12 & P12p & PmUG01_11050400 & Y19-S333 & 315 & Low & 6-cysteine protein & 126822 \\
\hline & P38 & & PmUG01_06016900 & M1-A310 & 310 & Low & $\begin{array}{l}\text { 6-cysteine protein, } \\
\text { putative }\end{array}$ & 126823 \\
\hline & $\mathrm{P} 41^{*}$ & & PmUG01_03015000 & Q23-D374 & 352 & Med & $\begin{array}{l}\text { 6-cysteine protein, } \\
\text { putative }\end{array}$ & 126824 \\
\hline & MSP3.10 & & PmUG01_06022000 & K24-1525 & 522 & High & $\begin{array}{l}\text { Merozoite surface } \\
\text { protein 3, putative }\end{array}$ & 126825 \\
\hline & MSP3.5 & & PmUG01_06022600 & N21-S562 & 542 & High & $\begin{array}{l}\text { Merozoite surface } \\
\text { protein 3, putative }\end{array}$ & 126826 \\
\hline & MSP7.1 & & PmUG01_12029500 & R25-1442 & 418 & High & $\begin{array}{l}\text { MSP7-like protein, } \\
\text { putative }\end{array}$ & 126827 \\
\hline & MSP7.4 & & PmUG01_12029900 & K22-V394 & 373 & High & $\begin{array}{l}\text { MSP7-like protein, } \\
\text { putative }\end{array}$ & 126828 \\
\hline \multirow[t]{12}{*}{ P. ovale } & CyRPA & & POVCU1_054880 & S20-D359 & 340 & Med & $\begin{array}{l}\text { Cysteine-rich protec- } \\
\text { tive antigen, putative, } \\
\text { SBS99762.1 }\end{array}$ & 126829 \\
\hline & GAMA & & PocGH01_05012100 & L22-S755 & 734 & High & $\begin{array}{l}\text { GPI-anchored microne- } \\
\text { mal antigen, putative }\end{array}$ & 126830 \\
\hline & MSP10* & & PocGH01_11036900 & Y27-S430 & 404 & High & $\begin{array}{l}\text { Merozoite surface pro- } \\
\text { tein 10, putative }\end{array}$ & 126831 \\
\hline & MSP4 & & PocGH01_04023000 & N26-\$209 & 184 & High & $\begin{array}{l}\text { Merozoite surface } \\
\text { protein 4, putative }\end{array}$ & 126832 \\
\hline & MSP5 & & PocGH01_04023100 & F20-S311 & 292 & High & $\begin{array}{l}\text { Merozoite surface } \\
\text { protein } 5 \text {, putative }\end{array}$ & 126833 \\
\hline & P12 & $P 12 p$ & PocGH01_11044100 & E23-S301 & 279 & Low & 6-cysteine protein & 126834 \\
\hline & P38 & & PocGH01_10033500 & K25-S332 & 308 & Low & $\begin{array}{l}\text { 6-cysteine protein, } \\
\text { putative }\end{array}$ & 126835 \\
\hline & $\mathrm{P} 41^{*}$ & & PocGH01_03012400 & E16-E375 & 360 & Med & $\begin{array}{l}\text { 6-cysteine protein, } \\
\text { putative }\end{array}$ & 126836 \\
\hline & MSP3.5* & & PocGH01_10038700 & K24-P480 & 457 & Med & $\begin{array}{l}\text { Merozoite surface } \\
\text { protein 3, putative }\end{array}$ & 126837 \\
\hline & MSP3.6 & & PocGH01_10038800 & K24-S732 & 709 & Low & $\begin{array}{l}\text { Merozoite surface } \\
\text { protein 3, putative }\end{array}$ & 126838 \\
\hline & MSP7.12 & & PocGH01_12028800 & K23-T441 & 419 & Med & $\begin{array}{l}\text { MSP7-like protein, } \\
\text { putative }\end{array}$ & 126839 \\
\hline & MSP7.8 & & PocGH01_12028400 & K23-T379 & 357 & Med & $\begin{array}{l}\text { MSP7-like protein, } \\
\text { putative }\end{array}$ & 126840 \\
\hline
\end{tabular}


Table 1 (continued)

\begin{tabular}{|c|c|c|c|c|c|c|c|c|}
\hline Sp. & $\begin{array}{l}\text { Official } \\
\text { nomenclature }\end{array}$ & Synonym/s & Accession number & $\begin{array}{l}\text { Region } \\
\text { expressed }\end{array}$ & $\begin{array}{l}\text { Length } \\
\text { (aa) }\end{array}$ & $\begin{array}{l}\text { Exp. } \\
\text { level }\end{array}$ & PlasmoDB, previous ID & $\begin{array}{l}\text { Addgene } \\
\text { ID }\end{array}$ \\
\hline \multirow[t]{9}{*}{ P. knowlesi } & CyRPA & & PKNH_0515800 & N23-E366 & 344 & Med & $\begin{array}{l}\text { Cysteine-rich protec- } \\
\text { tive antigen, putative, } \\
\text { PKH_052740 }\end{array}$ & 126841 \\
\hline & GAMA & PSOP9 & PKNH_1322900 & L21-S700 & 680 & Med & $\begin{array}{l}\text { GPI-anchored microne- } \\
\text { mal antigen, putative, } \\
\text { PKH_050210 }\end{array}$ & 126842 \\
\hline & MSP10* & & PKNH_1129800 & N27-S417 & 391 & High & $\begin{array}{l}\text { Merozoite surface } \\
\text { protein 10, putative, } \\
\text { PKH_112880 }\end{array}$ & 126940 \\
\hline & MSP4 & & PKNH_0414100 & D26-S182 & 157 & Med & $\begin{array}{l}\text { Merozoite surface } \\
\text { protein 4, putative, } \\
\text { PKH_041300 }\end{array}$ & 126843 \\
\hline & MSP5 & & PKNH_0414200 & $\mathrm{H} 22-\mathrm{S} 354$ & 333 & Med & $\begin{array}{c}\text { Merozoite surface pro- } \\
\text { tein 5,PKH_041310 }\end{array}$ & 126844 \\
\hline & $\mathrm{P} 12^{*}$ & & PKNH_1137300 & F24-S323 & 300 & Med & $\begin{array}{l}\text { 6-cysteine protein, } \\
\text { PKH_113620 }\end{array}$ & 126845 \\
\hline & P38* & & PKNH_1025600 & K3-S367 & 365 & Low & $\begin{array}{l}\text { 6-cysteine protein, } \\
\text { PKH_102490 }\end{array}$ & 126846 \\
\hline & P41 & & PKNH_0303000 & E22-E393 & 372 & High & $\begin{array}{l}\text { 6-cysteine protein, } \\
\text { PKH_030970 }\end{array}$ & 126847 \\
\hline & P92 & & PKNH_1107200 & D24-P857 & 834 & Med & $\begin{array}{l}\text { 6-cysteine protein, } \\
\text { PKH_110660 }\end{array}$ & 126848 \\
\hline
\end{tabular}

Protein expression plasmids encoding the named genes from the five Plasmodium species that infect humans are detailed. 'Region expressed' refers to the ectodomain regions between the predicted signal sequence peptide and the transmembrane or GPI anchor sequence (if present). Expression levels are given as a guide only given the significant batch-to-batch variability observed using this approach and grouped into 'high' (between 5 and $50 \mu \mathrm{g} / \mathrm{mL}$ ), 'medium' ( $0.5-5 \mu \mathrm{g} / \mathrm{mL}$ ) and 'low' (0.005-0.5 $\mu \mathrm{g} / \mathrm{mL})$. Those proteins selected for further serological analysis are marked with an asterisk

levels (Fig. 1b); MSP4 was not selected because although immunoreactive, was repeatedly expressed poorly. Using the same criteria, the P. ovale proteins P41, MSP3.5 and MSP10 were selected (Fig. 1b). To identify the most suitable antigens for P. knowlesi, the immunoreactivity to the panel of proteins was tested using sera derived from 10 Malaysian patients with PCR-confirmed single P. knowlesi infections, and P12, P38 and MSP10 were chosen (Fig. 1c). From these experiments, a panel of 22 proteins were selected that contained 7 proteins from $P$. falciparum, 6 from $P$. vivax, and 3 from each of $P$. malariae, P. ovale and P. knowlesi (Table 1).

Using this panel of 22 immunoreactive proteins, the antibody responses to a specific antigen from a particular Plasmodium species were examined to determine whether there was any detectable cross-reactivity with antigens from other species. Ideally, this would make use of sera from human patients which have diagnosed mono-infections for each Plasmodium species. To increase the chances that patients had been exposed to a single species, serum samples were obtained from patients of European origin who had contracted malaria by visiting malaria endemic regions and in which the infecting Plasmodium species had been confirmed by
PCR. For the more common parasites, a reasonable number of samples were obtained: $P$. falciparum $(n=26), P$. vivax $(n=17)$, but access to serum samples of travellers' malaria with diagnosed infections for the rarer parasites was limited: $P$. ovale $(n=7), P$. malariae $(n=3)$. For $P$. knowlesi, responses in the 10 Malaysian patients with PCR-confirmed single P. knowlesi infections were used. These patient sera were screened against the panel of all 22 antigens from five Plasmodium species. Two patients exhibited a broad reactivity to antigens from two species: a Swedish traveller diagnosed with $P$. vivax but whose serum exhibited broad reactivity across antigens from three Plasmodium species (P. vivax, $P$. falciparum, $P$. knowlesi) and one of the Malaysian patients diagnosed with $P$. knowlesi but whose serum reacted strongly with four out of six $P$. vivax antigens; this patient was subsequently identified as a migrant worker from Indonesia where $P$. vivax is endemic. Because it is likely that these patients had prior exposure to another Plasmodium species other than their diagnosed infection, these patients were removed from this analysis. The pairwise correlation of the responses to each of the antigens in the panel was examined in the remaining patients (Fig. 2a). For P. falciparum and P. vivax, where more samples were 


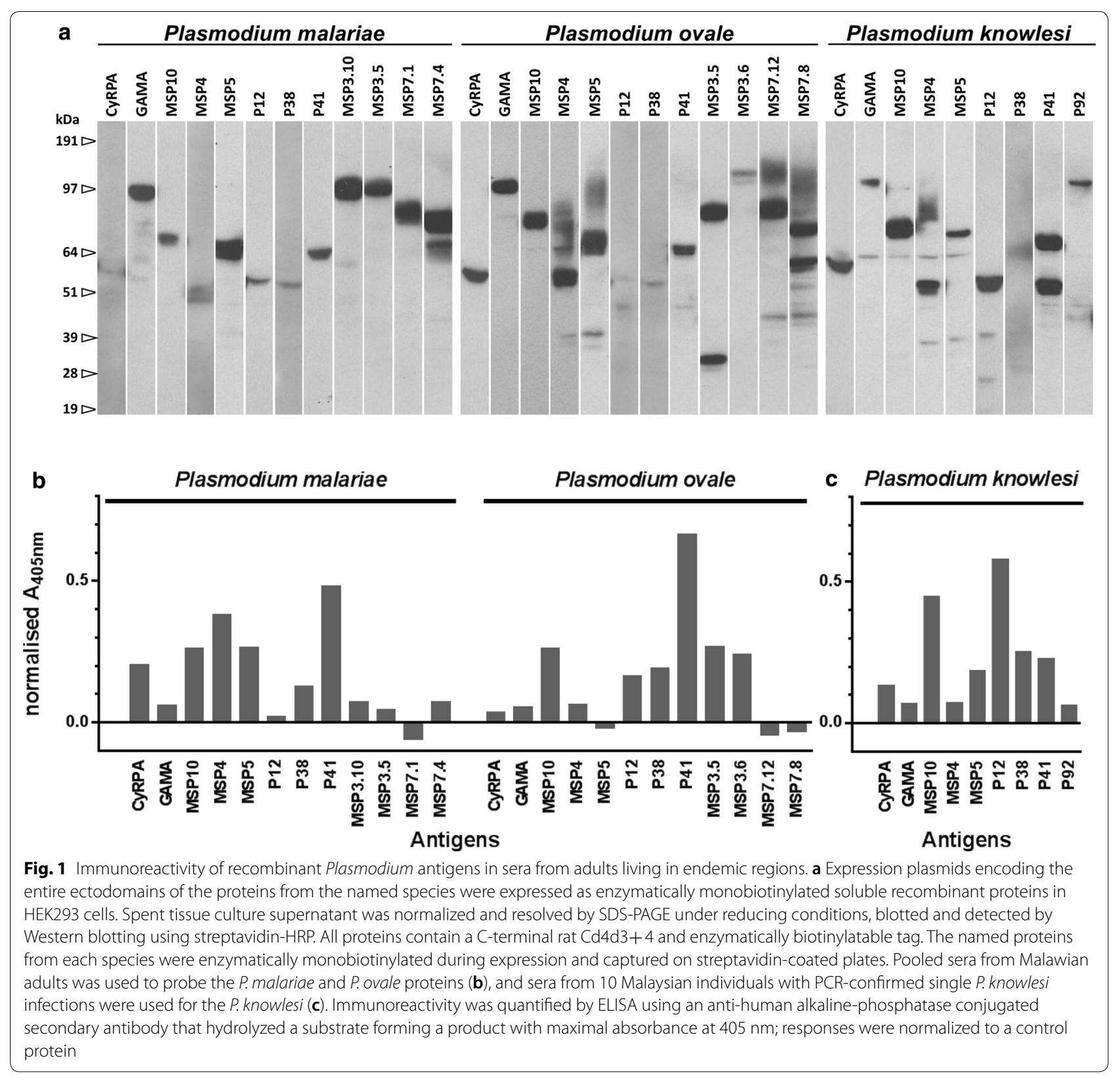

available, the antibody responses to most antigens within that species were positively correlated, as expected (Fig. 2a). There were two antigens that showed significant cross-reactivity between their orthologues: the $\mathrm{HPzz}$ proteins from $P$. falciparum and P. vivax, and MSP5 from $P$. vivax and $P$. malariae. Furthermore, responses to $P$. malariae P41 correlated with $P$. ovale MSP3.5. This suggested that responses to these proteins may not be suitable to diagnose exposure for these species, and so were omitted from further analyses. There was no evidence that sequence identity between orthologous proteins correlated with cross reactivity. For example, the amino acid sequence identity between $P$. falciparum and $P$. vivax
HPzz proteins was only 39\%, and yet responses showed strong evidence of cross-reactivity (Fig. 2b). By contrast, the sequence identity between $P$. knowlesi and $P$. vivax P12 was much higher (72\%), and yet patient responses showed little evidence of cross-reaction (Fig. 2c). While this analysis must come with the caveat that the number of serum samples from travellers with malaria is limited, especially for the rarer parasites, $P$. ovale and $P$. malariae, antigens that appeared potentially cross-reactive between species were excluded, and this apparent cross-reactivity did not correlate with sequence identity between the orthologous proteins. 


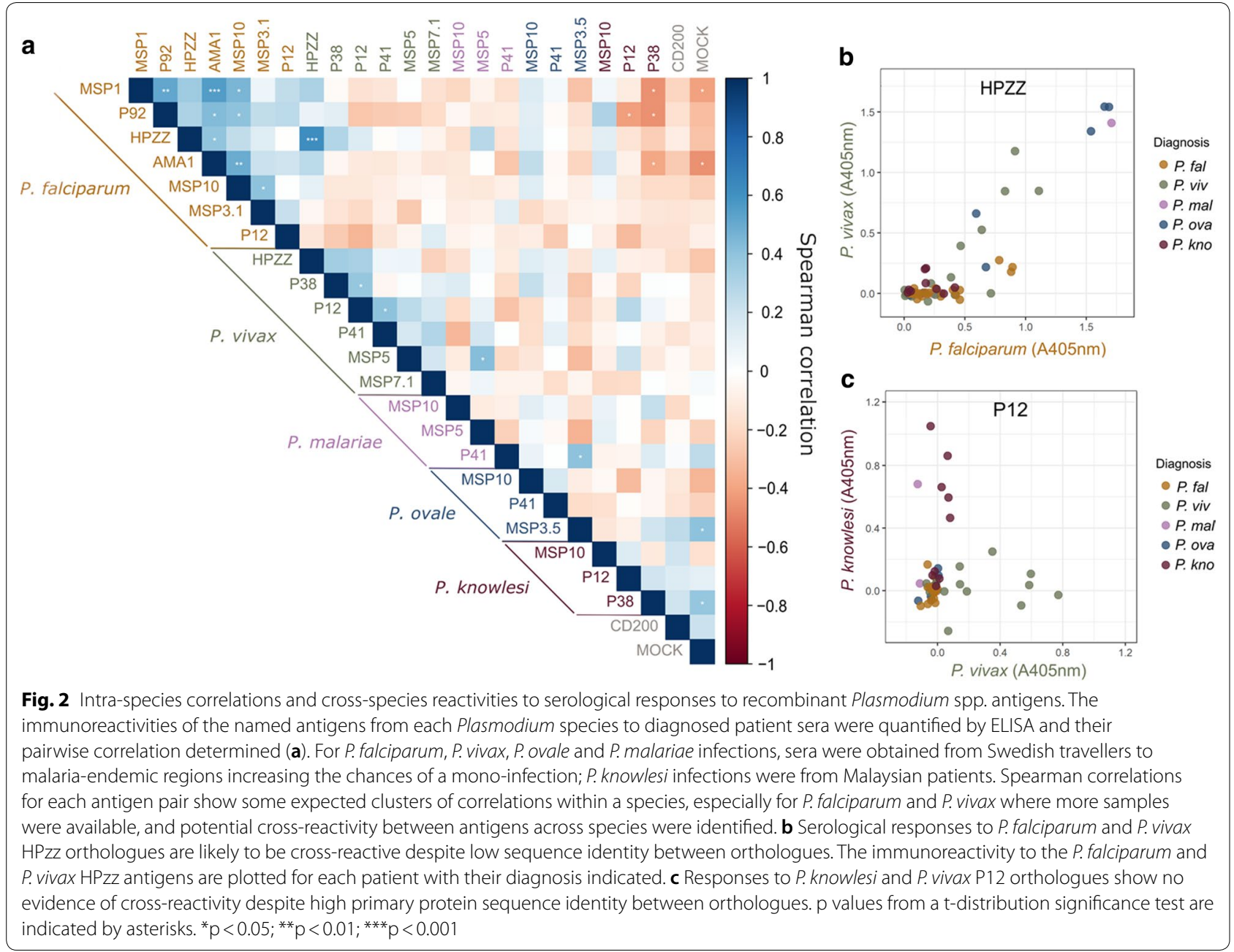

To determine whether the panel of proteins could be used in serological assays to establish exposure to different human-infective Plasmodium species, a larger panel of serum samples from individuals with diagnosed infections that corresponded to both ongoing acute cases of imported malaria $(n=81)$, and endemic malaria from a cohort of Tanzanian adults $(n=21)$ were tested. For $P$. knowlesi, sera from the 10 patients diagnosed with $P$. knowlesi infections from Kapit Hospital in Malaysian Borneo were used. The antibody responses between the different sources were investigated by segregating the samples into three different categories: travellers of European origin $(n=53)$, travellers with an origin in a malaria-endemic area $(n=28)$, and individuals residing in an endemic area $(n=31)$. The responses to each antigen corresponding to the species diagnosis was plotted within each category, and while differences between the different patient groupings were observed on a per antigen basis (Additional file 2: Fig. S1a), when averaged across the panel, no systematic differences were observed
(Additional file 2: Fig. S1b). The converse analysis was performed whereby the responses to the antigens corresponding to the species other than that in the diagnosis were plotted, and again segregated according to the different categories. As expected, there was little response across the antigen panel, although it was clear that the Tanzanian endemic samples showed evidence of exposure to other species other than the one for which they had a diagnosed ongoing infection, especially for $P$. falciparum, but also P. ovale (Additional file 2: Fig. S1c). Immunoreactivity to both HPzz orthologues from both $P$. falciparum and P. vivax and PfMSP1 were observed which suggested antigen cross-reactivity, as had been already established for HPzz (Fig. 2), or, in the case of PfMSP1, there was a higher background signal, including from the uninfected controls (Additional file 2: Fig. S1c).

A logistic-regression classifier was trained to combine the immunoreactivities to the antigens from each species into a prediction of prior exposure. Sera from both travellers and individuals living in endemic regions for each 
species were used together with unexposed control samples and iteratively and randomly split 50:50 into training and testing sets, with approximately equal representation of diagnoses for each species. For each Plasmodium species, a separate model was trained to assign a binary outcome indicating if a patient was infected by that species or not. A score near zero indicates that the model assigns a low probability of the patient being infected with the given Plasmodium species, while scores near one indicate likely infections. Because patients from endemic regions are very likely to have had prior exposure to species other than the one they were actively diagnosed with, the positive training set was defined as patients only with a confirmed species diagnosis by microscopy or PCR.

The performance of each diagnostic model was evaluated, and the random sampling procedure into training and testing sets was iteratively repeated. To quantify the models' performance, the averaged receiver operating characteristic (ROC) curves for predicting exposure to each species (Fig. 3a) was first calculated. Moderate predictive power (area under the curve $(\mathrm{AUC}) \geq 0.7$ ) was achieved for all species, and especially for $P$. vivax,

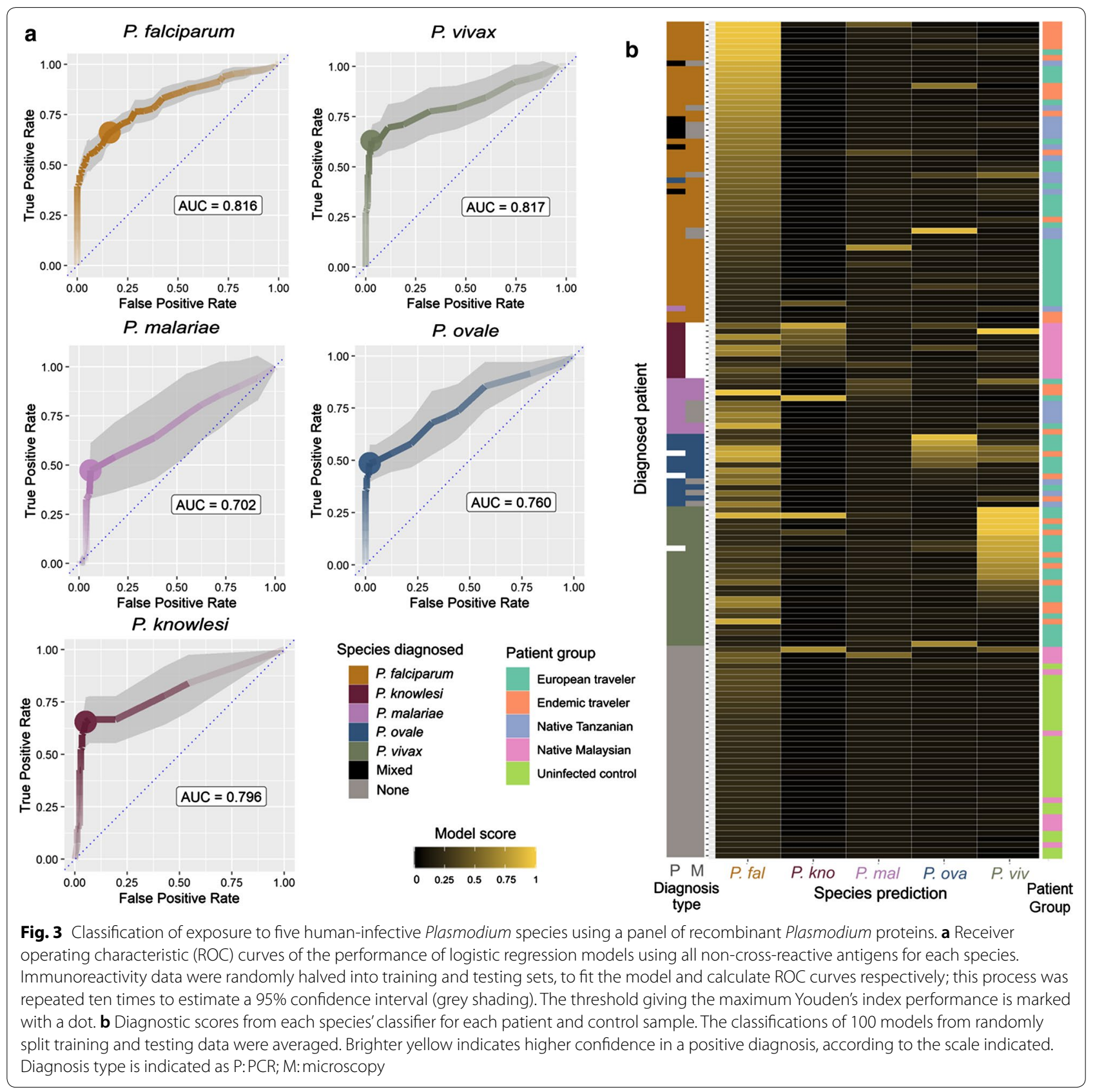


P. falciparum and P. knowlesi where exposure could be predicted with good accuracy (AUC $\geq 0.8$ ). The relatively small number of available diagnosed samples for the rarer parasites $P$. ovale $(n=18)$ and $P$. malariae $(n=17)$, limited the performance of their classifiers, as well as in the case of $P$. malariae, the reliance on just a single antigen (PmMSP10) after removing those that showed evidence of cross-reactivity.

Diagnostic models were used to determine a likelihood of prior exposure of the patients to each of the different Plasmodium species (Fig. 3b). In general, the models returned a positive prediction for the parasite species that agreed with the diagnosis, and the sera from unexposed controls being negative. The classifiers performed less well on those patients with co-infections and those diagnosed by PCR rather than microscopy (Fig. 3b). As expected, several individuals showed strong evidence of prior exposure to a different Plasmodium parasite species other than the one for which they were positively diagnosed. For example, some individuals positively diagnosed with $P$. ovale infections, also showed evidence of prior exposure to $P$. falciparum and $P$. vivax.

Following the encouraging performance of the antigen panel in diagnosing infections by $P$. knowlesi, a larger number of serum samples comprising 50 P. knowlesi malaria patients and 66 uninfected controls from the same region in Malaysia were evaluated. Using the logistic regression model to combine the responses against all three antigens (PkMSP10, PkP12, PkP38), P. knowlesi infections could be identified with high confidence (AUC > 91\%) (Fig. 4a). If a threshold is set to a model score of above 0.5 , then $82 \%$ of P. knowlesi-infected patients are correctly diagnosed at a false-positive rate of 3\% (Fig. 4b).

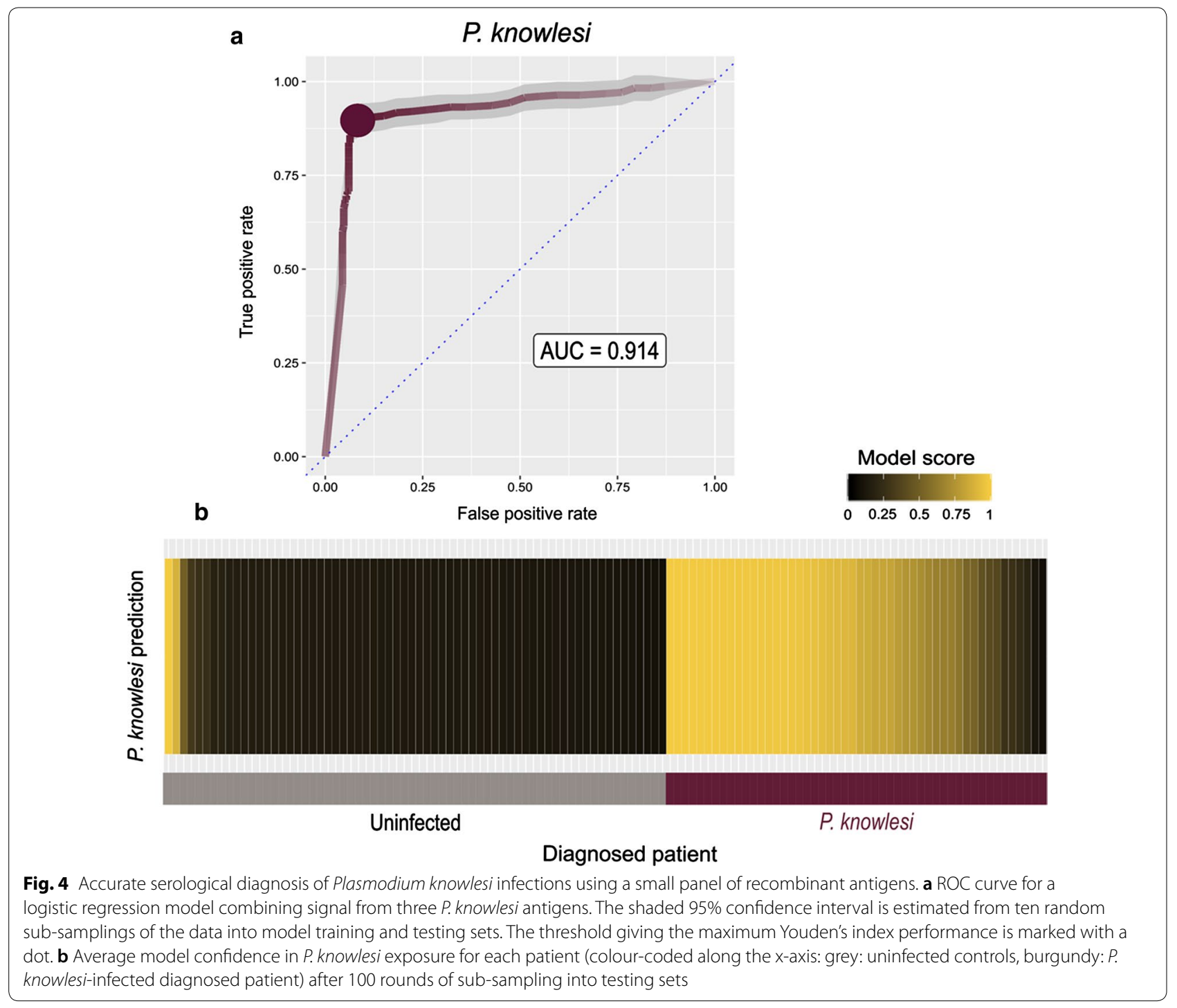




\section{Discussion}

Malaria continues to be a significant global health problem although cases have dropped in recent years due to the deployment of effective intervention measures [1]. Spurred on by these successes, the malaria control policies of governments and international agencies are moving towards the goal of eradication, which will require more sensitive detection and tracking of the different species in endemic regions. The application of serological screening may have an increasingly important role as transmission declines because the longevity of antibody responses should provide a historical record of exposure rather than being limited to the detection of current infections [36]. In areas of declining transmission, where there are increasing incidences of sub-microscopic infection, models using data from serological surveillance have been shown to provide precise estimates of parasite transmission [10]. Because of the asexual amplification of malaria parasites in the blood, those antigens expressed during these blood stages are likely to be good choices for diagnostic antigens because they are known to be particularly immunodominant. Several serological assays have been developed to detect exposure to Plasmodium parasites although few have attempted to distinguish infections by the different species [37]. This has mainly been due to the lack of a genome sequence from the two parasites, $P$. ovale and $P$. malariae, making the cloning and expression of many candidate proteins from these species difficult. Recent sequencing of $P$. ovale and $P$. malariae [23, 24] has now permitted the expression of several candidate proteins from these parasites. Here, a panel of antigens was compiled that are likely to be expressed by the blood stages using the recent availability of the genome sequences of all five main species of Plasmodium parasites that infect humans, together with the approach of expressing extracellular parasite proteins in a functionally active form using a mammalian expression system. After removing those proteins which showed evidence of cross-reactive responses, it was found that immunoreactivity to the antigens from $P$. ovale, (MSP10, P41), P. malariae (MSP10) and P. knowlesi (MSP10, P12, P38), together with logistic regression modelling had moderate power to predict prior exposure to these species, and performed especially well for P. knowlesi.

Expressing Plasmodium proteins is known to be technically challenging, possibly due to the unusual codon bias or highly repetitive amino acid sequences often found in Plasmodium proteins [15, 38]. Most studies expressing recombinant antigens for serological assays use prokaryotic expression systems (especially Escherichia coli) or cell-free systems, such as wheat germ extracts [39-41]. While these expression systems have the advantages of being cost effective, high yielding and widely available, they may not be suitable for expressing extracellular proteins because they usually require a reducing environment which would interfere with the formation of structurally critical disulfide bonds [16, 42]. While protein refolding procedures can be used, they are usually complex, time consuming, and have uncertain outcomes which are often hard to determine if they have been successful [43]. When used for serological screening, proteins that do not adopt the native conformation are unlikely to be useful in detecting antibodies that recognise conformational epitopes, potentially reducing sensitivity. By using a mammalian expression system to produce Plasmodium proteins, it was previously shown that these recombinant proteins can retain conformational epitopes, and shown that for the majority of antigens, a large fraction of the immunoreactivity to sera from Plasmodium-exposed patients is heat labile, demonstrating the benefit of using proteins that retain native folding for serological assays [16]. Plasmodium blood-stage proteins expressed using this approach have been useful in sero-epidemiological studies for P. falciparum [12, 44] and P. vivax [8]. One likely consequence of using conformational epitopes in serological assays, which are aimed at distinguishing between species, is that the percentage sequence identity between orthologous proteins is unlikely to be a reliable indicator of cross-reactivity. In the current study, a likely cross-reactivity was observed between the P. falciparum and P. vivax orthologues of the HPzz protein between patients diagnosed with these parasites, despite there being only $\sim 40 \%$ amino acid shared sequence identity. By contrast, very little cross-reactivity was observed in patient serological responses between the P. vivax and P. knowlesi P12 orthologues which are relatively well conserved, sharing $>70 \%$ amino acid sequence identity. One likely possibility is that the informative epitopes for these proteins are mainly composed of the precise arrangement of solvent-exposed amino acids, making overall linear sequence identity less important, whereas repeats of just a few amino acids, for example the repetitive "NANP" motifs in the circumsporozoite protein can be highly immunogenic [45].

A panel of three recombinant antigens (PkMSP10, PkP12, PkP38), was found to accurately detect exposure to $P$. knowlesi using a serological assay. This compares well with a recent study where the authors also selected candidates that were orthologous to known immunoreactive proteins in $P$. falciparum, and selected four antigens that were associated with $P$. knowlesi exposure [46]. Together, these studies will contribute towards the further development of accurate serological assays for this parasite which is becoming an increasing 
public health concern in Southeast Asia, particularly in Malaysia where it is now replacing $P$. falciparum and $P$. vivax as the major cause of malaria $[47,48]$.

The use of plasma from native Europeans who had contracted travellers' malaria and had been diagnosed accurately increased the chances that they had only been exposed to a single species which was useful for establishing which responses to different antigens might be cross reactive. However, accessing large numbers of these samples was difficult, especially for the rarer parasites, $P$. malariae and $P$. ovale, and therefore it is not certain that these patients had been previously exposed to other species of Plasmodium. Together with other assays that have been described for serological diagnosis of Plasmodium infections [37, 46, 49], this panel of proteins will contribute to an assay that will be a useful tool for establishing prior exposure to different species of Plasmodium parasites. Surveying more patient samples in longitudinal cohorts in a variety of transmission settings will be necessary to establish whether the responses to the proteins described here are different in patients living in different endemic regions, and vary according to the age and exposure of the patient.

\section{Conclusions}

The recent availability of genome sequences for Plasmodium parasites was used to create a panel of recombinant proteins corresponding to immunoreactive blood stage proteins from five human-infective Plasmodium species. This panel of proteins will provide a basis for developing serological assays to determine exposure to the different species of parasite for serological surveillance and diagnostics.

\section{Supplementary information}

Supplementary information accompanies this paper at https://doi. org/10.1186/s12936-020-3111-5.

\begin{abstract}
Additional file 1: Datasets used for the analysis. The ELISA absorbance values used for the data analysis are provided as separate spreadsheets. Patient groups where applicable are numbered: 1 -European traveller; 2 and 3-Imported malaria; 4-Native Tanzanian; 5-Control group; 6Native Malaysian.

Additional file 2: Fig. S1. Antibody responses to the Plasmodium antigen panel across sample groups. (a) Immunoreactivity to antigens for patients diagnosed with an infection from the corresponding species, grouped by patient background. (b) Average immunoreactivity to antigens for each species in patients diagnosed for that species. (c) Background immunoreactivity to antigens for patients not diagnosed with the corresponding Plasmodium species.
\end{abstract}

\section{Abbreviations}

AUC: area under curve; ELISA: enzyme-linked immunosorbent assay; HEK: human embryonic kidney; PCR: polymerase chain reaction; RDT: rapid diagnostic test; ROC: receiver operator characteristic; SDS PAGE: sodium dodecyl sulfate polyacrylamide gel electrophoresis.

\section{Acknowledgements}

We thank Gavin Rutledge for providing sequences for the $P$. ovale and $P$. malariae proteins, the Medical Laboratory Technologists, nursing and medical staff at Kapit Hospital for sera collections from malaria patients and uninfected controls, and staff at Karolinska University Hospital and Nyamisati Malaria Research project.

\section{Authors' contributions}

NMS performed all protein expression and ELISAs; JS performed data analysis and modelling; KAK organized Malaysian sera collections and performed diagnostic PCR under supervision of $B S ; A F, V Y$ and $B N$ were responsible for cohorts in Sweden and Tanzania; VY, MVH and MA organized sera collections and performed diagnostic PCR under the guidance of AF; BS provided sera from $P$. knowlesi-infected patients. GJW conceived and managed the study together with NMS, AF and VY. GJW wrote the manuscript with input from JS, $B S, A F$, and NMS. All authors read and approved the final manuscript.

\section{Funding}

This research was supported by the Wellcome Trust Grant (206194), A Churchill Scholarship to JS, Swedish Research Council, Medicine and Health and Development Research Project grants (AF), and Universiti Malaysia Sarawak Top Down Grant (FA052000-0706-0002).

\section{Availability of data and materials}

All plasmid protein expression constructs are available from the Addgene resource provider (www.addgene.org). The datasets used and/or analysed during the current study are available in Additional file 1.

\section{Ethics approval and consent to participate}

Ethical approval was granted by the Ethical Review board of the National Institute for Medical Research in Tanzania, the Regional Ethical Review Board in Stockholm, Sweden (Dnr. 00-084, 2012/1151-32, 2006/893-31/4, 2018/235432), the Medical Research and Ethics Committee of the Ministry of Health, Malaysia and the Medical Ethics Committee, and Faculty of Medicine and Health Sciences, Universiti Malaysia Sarawak.

\section{Consent for publication}

Not applicable.

\section{Competing interests}

The authors declare that they have no competing interests.

\section{Author details}

${ }^{1}$ Cell Surface Signalling Laboratory, Wellcome Sanger Institute, Cambridge, UK. ${ }^{2}$ Malaria Research Centre, Universiti Malaysia Sarawak, Kota Samarahan, Sarawak, Malaysia. ${ }^{3}$ Division of Infectious Diseases, Department of Medicine Solna, Karolinska Institutet, Stockholm, Sweden. ${ }^{4}$ Department of Parasitology and Medical Entomology, Muhimbili University of Health and Allied Sciences, Dar es Salaam, Tanzania. ${ }^{5}$ Department of Women's and Children's Health, International Maternal and Child Health (IMCH), Uppsala University, Uppsala, Sweden. ${ }^{6}$ Department of Infectious Diseases, Karolinska University Hospital, Stockholm, Sweden.

Received: 9 July 2019 Accepted: 8 January 2020

Published online: 17 January 2020

\section{References}

1. WHO. World Malaria Report. Geneva: World Health Organization; 2018.

2. Adams JH, Mueller I. The biology of Plasmodium vivax. Cold Spring Harb Perspect Med. 2017;7:a025585.

3. Singh B, Daneshvar C. Human infections and detection of Plasmodium knowlesi. Clin Microbiol Rev. 2013;26:165-84.

4. Hemingway J, Shretta R, Wells TN, Bell D, Djimde AA, Achee N, et al. Tools and strategies for malaria control and elimination: what do we need to achieve a grand convergence in malaria? PLoS Biol. 2016;14:e1002380. 
5. Corran P, Coleman P, Riley E. Drakeley C serology: a robust indicator of malaria transmission intensity? Trends Parasitol. 2007;23:575-82.

6. Yman V, White MT, Asghar M, Sundling C, Sonden K, Draper SJ, et al. Antibody responses to merozoite antigens after natural Plasmodium falciparum infection: kinetics and longevity in absence of re-exposure. BMC Med. 2019;17:22

7. Drakeley CJ, Corran PH, Coleman PG, Tongren JE, McDonald SL, Carneiro I, et al. Estimating medium- and long-term trends in malaria transmission by using serological markers of malaria exposure. Proc Natl Acad Sci USA. 2005;102:5108-13.

8. Franca CT, Hostetler JB, Sharma S, White MT, Lin E, Kiniboro B, et al. An antibody screen of a Plasmodium vivax antigen library identifies novel merozoite proteins sssociated with clinical protection. PLoS Negl Trop Dis. 2016;10:e0004639.

9. Helb DA, Tetteh KK, Felgner PL, Skinner J, Hubbard A, Arinaitwe E, et al. Novel serologic biomarkers provide accurate estimates of recent Plasmodium falciparum exposure for individuals and communities. Proc Natl Acad Sci USA. 2015:112:e4438-47.

10. Yman V, White MT, Rono J, Arca B, Osier FH, Troye-Blomberg M, et al. Antibody acquisition models: a new tool for serological surveillance of malaria transmission intensity. Sci Rep. 2016;6:19472.

11. Crompton PD, Kayala MA, Traore B, Kayentao K, Ongoiba A, Weiss GE, et al. A prospective analysis of the $\mathrm{Ab}$ response to Plasmodium falciparum before and after a malaria season by protein microarray. Proc Natl Acad Sci USA. 2010;107:6958-63.

12. Osier FH, Mackinnon MJ, Crosnier C, Fegan G, Kamuyu G, Wanaguru M, et al. New antigens for a multicomponent blood-stage malaria vaccine. Sci Transl Med. 2014;6:247ra102.

13. Allain JP. Malaria and transfusion: a neglected subject coming back to the forefront. Clin Infect Dis. 2010;51:1199-200.

14. Forsstrom B, Axnas BB, Rockberg J, Danielsson H, Bohlin A. Uhlen M Dissecting antibodies with regards to linear and conformational epitopes. PLOS ONE. 2015;10:e0121673.

15. Birkholtz LM, Blatch G, Coetzer TL, Hoppe HC, Human E, Morris EJ, et al. Heterologous expression of plasmodial proteins for structural studies and functional annotation. Malar J. 2008;7:197.

16. Crosnier C, Wanaguru M, McDade B, Osier FH, Marsh K, Rayner JC, et al. A library of functional recombinant cell-surface and secreted $P$. falciparum merozoite proteins. Mol Cell Proteomics. 2013;12:3976-86.

17. Zenonos ZA, Rayner JC. Wright GJ Towards a comprehensive Plasmodium falciparum merozoite cell surface and secreted recombinant protein library. Malar J. 2014:13:93.

18. Hostetler JB, Sharma S, Bartholdson SJ, Wright GJ, Fairhurst RM. Rayner JC A Library of Plasmodium vivax recombinant merozoite proteins reveals new vaccine candidates and protein-protein interactions. PLoS Negl Trop Dis. 2015;9:e0004264.

19. Taylor TE, Molyneux ME, Wirima JJ, Borgstein A, Goldring JD, Hommel $M$. Intravenous immunoglobulin in the treatment of paediatric cerebral malaria. Clin Exp Immunol. 1992;90:357-62.

20. Vafa Homann M, Emami SN, Yman V, Stenstrom C, Sonden K, Ramstrom $\mathrm{H}$, et al. Detection of malaria parasites after treatment in travelers: a 12-months longitudinal study and statistical modelling analysis. EBioMed 2017:25:66-72

21. Yman V, Wandell G, Mutemi DD, Miglar A, Asghar M, Hammar U, et al. Persistent transmission of Plasmodium malariae and Plasmodium ovale species in an area of declining Plasmodium falciparum transmission in eastern Tanzania. PLoS Negl Trop Dis. 2019;13:e0007414.

22. Pain A, Bohme U, Berry AE, Mungall K, Finn RD, Jackson AP, et al. The genome of the simian and human malaria parasite Plasmodium knowlesi. Nature. 2008:455:799-803.

23. Ansari HR, Templeton TJ, Subudhi AK, Ramaprasad A, Tang J, Lu F, et al. Genome-scale comparison of expanded gene families in Plasmodium ovale wallikeri and Plasmodium ovale curtisi with Plasmodium malariae and with other Plasmodium species. Int J Parasitol. 2016:46:685-96.

24. Rutledge GG, Bohme U, Sanders M, Reid AJ, Cotton JA, Maiga-Ascofare $\mathrm{O}$, et al. Plasmodium malariae and $P$. ovale genomes provide insights into malaria parasite evolution. Nature. 2017;542:101-4.

25. Krogh A, Larsson B, von Heijne G. Sonnhammer EL Predicting transmembrane protein topology with a hidden Markov model: application to complete genomes. J Mol Biol. 2001;305:567-80.
26. Petersen TN, Brunak S, von Heijne G, Nielsen H. SignalP 4.0: discriminating signal peptides from transmembrane regions. Nat Methods. 2011:8:785-6.

27. Crosnier C, Staudt N. Wright GJ A rapid and scalable method for selecting recombinant mouse monoclonal antibodies. BMC Biol. 2010;8:76.

28. Sun Y, Gallagher-Jones M, Barker C. Wright GJ A benchmarked protein microarray-based platform for the identification of novel low-affinity extracellular protein interactions. Anal Biochem. 2012;424:45-53.

29. Durocher Y, Perret S. Kamen A High-level and high-throughput recombinant protein production by transient transfection of suspensiongrowing human 293-EBNA1 cells. Nucleic Acids Res. 2002;30:E9.

30. Loignon M, Perret S, Kelly J, Boulais D, Cass B, Bisson L, et al. Stable high volumetric production of glycosylated human recombinant IFNalpha2b in HEK293 cells. BMC Biotechnol. 2008;8:65.

31. Kerr JS, Wright GJ. Avidity-based extracellular interaction screening (AVEXIS) for the scalable detection of low-affinity extracellular receptor-ligand interactions. J Vis Exp. 2012;61:e3881

32. Fischer JE, Bachmann $L M$. Jaeschke $R A$ readers' guide to the interpretation of diagnostic test properties: clinical example of sepsis. Intensiv Care Med. 2003:29:1043-51.

33. Mandrekar JN. Receiver operating characteristic curve in diagnostic test assessment. J Thorac Oncol. 2010;5:1315-6.

34. Grau J, Grosse I. Keilwagen J PRROC: computing and visualizing precision-recall and receiver operating characteristic curves in R. Bioinformatics. 2015;31:2595-7.

35. Koita OA, Sangare L, Sango HA, Dao S, Keita N, Maiga M, et al. Effect of seasonality and ecological factors on the prevalence of the four malaria parasite species in northern Mali. J Trop Med. 2012:2012:367160

36. Wipasa J, Suphavilai C, Okell LC, Cook J, Corran PH, Thaikla K, et al. Long-lived antibody and B cell memory responses to the human malaria parasites, Plasmodium falciparum and Plasmodium vivax. PLoS Pathog. 2010;6:e1000770.

37. Muerhoff AS, Birkenmeyer LG, Coffey R, Dille BJ, Barnwell JW, Collins $W E$, et al. Detection of Plasmodium falciparum, P. vivax, P. ovale, and $P$. malariae merozoite surface protein 1-p19 antibodies in human malaria patients and experimentally infected nonhuman primates. Clin Vaccine Immunol. 2010;17:1631-8.

38. Mehlin C, Boni E, Buckner FS, Engel L, Feist T, Gelb MH, et al. Heterologous expression of proteins from Plasmodium falciparum: results from 1000 genes. Mol Biochem Parasitol. 2006;148:144-60.

39. Arumugam TU, Ito D, Takashima E, Tachibana M, Ishino T, Torii M, et al. Application of wheat germ cell-free protein expression system for novel malaria vaccine candidate discovery. Expert Rev Vaccines. 2014;13:75-85.

40. Guerra AP, Calvo EP, Wasserman M, Chaparro-Olaya J. Production of recombinant proteins from Plasmodium falciparum in Escherichia coli. Biomedica. 2016;36:97-108

41. Travassos MA, Niangaly A, Bailey JA, Ouattara A, Coulibaly D, Lyke KE, et al. Children with cerebral malaria or severe malarial anaemia lack immunity to distinct variant surface antigen subsets. Sci Rep. 2018:8:6281.

42. Vedadi M, Lew J, Artz J, Amani M, Zhao Y, Dong A, et al. Genome-scale protein expression and structural biology of Plasmodium falciparum and related Apicomplexan organisms. Mol Biochem Parasitol. 2007:151:100-10.

43. Yamaguchi H. Miyazaki M refolding techniques for recovering biologically active recombinant proteins from inclusion bodies. Biomolecules. 2014:4.235-51.

44. Kamuyu G, Tuju J, Kimathi R, Mwai K, Mburu J, Kibinge N, et al. KILchip v1.0: a novel Plasmodium falciparum merozoite protein microarray to facilitate malaria vaccine candidate prioritization. Front Immunol. 2018;9:2866.

45. Kisalu NK, Idris AH, Weidle C, Flores-Garcia Y, Flynn BJ, Sack BK, et al. A human monoclonal antibody prevents malaria infection by targeting a new site of vulnerability on the parasite. Nat Med. 2018;24:408-16.

46. Herman LS, Fornace K, Phelan J, Grigg MJ, Anstey NM, William T, et al. Identification and validation of a novel panel of Plasmodium knowlesi biomarkers of serological exposure. PLoS Negl Trop Dis. 2018;12:e0006457. 
47. Moyes CL, Henry AJ, Golding N, Huang Z, Singh B, Baird JK, et al. Defining the geographical range of the Plasmodium knowlesi reservoir. PLoS Negl Trop Dis. 2014;8:e2780.

48. Cooper DJ, Rajahram GS, William T, Jelip J, Mohammad R, Benedict J, et al. Plasmodium knowlesi malaria in Sabah, Malaysia, 2015-2017: ongoing increase in incidence despite near-elimination of the human-only Plasmodium species. Clin Infect Dis. 2019. https://doi.org/10.1093/cid/ciz237.

49. Rodrigues MH, Cunha MG, Machado RL, Ferreira OC Jr, Rodrigues MM

Soares IS Serological detection of Plasmodium vivax malaria using recombinant proteins corresponding to the 19-kDa C-terminal region of the merozoite surface protein-1. Malar J. 2003;2:39.

\section{Publisher's Note}

Springer Nature remains neutral with regard to jurisdictional claims in published maps and institutional affiliations.
Ready to submit your research? Choose BMC and benefit from:

- fast, convenient online submission

- thorough peer review by experienced researchers in your field

- rapid publication on acceptance

- support for research data, including large and complex data types

- gold Open Access which fosters wider collaboration and increased citations

- maximum visibility for your research: over $100 \mathrm{M}$ website views per year

At BMC, research is always in progress.

Learn more biomedcentral.com/submissions 